\title{
A Cost-Performance Model for Ground-Based Optical Communications Receiving Telescopes
}

\author{
J. R. Lesh and D. L. Robinson \\ Communications Systems Research Section
}

\begin{abstract}
An analytical cost-performance model for a ground-based optical communications receiving telescope is presented. The model considers costs of existing telescopes as a function of diameter and field of view. This, coupled with communication performance as a function of receiver diameter and field of view, yields the appropriate telescope cost versus communication performance curve.
\end{abstract}

\section{Introduction}

At present, there is much interest in pursuing optical frequencies for deep space communication. Some of the advantages gained through the use of optical communication are: (1) higher data rate communication, (2) smaller size and mass components on the spacecraft compared to equivalent performance radio frequency systems (due to shorter wavelengths), and (3) precise navigational tracking of spacecraft against the stellar background with a single optical receiving station. When considering these advantages, one must also consider the cost of such a system and compare it to the corresponding quantities for other methods of providing equivalent service.

A detailed cost model of an entire deep space optical communication system is very difficult to create. It involves not only the spacecraft and Earth reception ends of the link, but the infrastructures which operate the overall system as well. To build such a model requires a concentrated effort on a number of individual ingredients. This article concentrates on one of those ingredients.
The most desirable location for an optical receiving station is in Earth orbit. However, in all likelihood a ground-based station will both proceed as well as augment an orbiting station. To understand the cost of a ground-based station one must consider also a number of elements. These include the telescope with its mount and pointing control, the dome or protective structure, site preparation, focal plane optics or electronics, control room electronics, ground communications links and the necessary software. Furthermore, recurring costs like maintenance, operations, utilities and spares are also important to consider.

This article considers one of the above elements of a groundbased station: the optical telescope. ${ }^{1}$ This element is believed to be one of the major cost ingredients for such a station, and the size and quality of the telescope have a bigger impact on performance than any other station elements.

\footnotetext{
${ }^{1}$ Telescope costs include costs associated with telescope mount and telescope pointing control.
} 
The general approach to this analysis is as follows: An analytical expression which describes telescope cost as a function of diameter and telescope resolution (determined by the surface quality of the primary and later equated to detector field of view) will first be determined. In order to relate telescope cost to optical communication performance, communication performance is considered as a function of telescope diameter and detector field of view. These two results are coupled together to yield telescope cost as a function of communication performance with telescope diameter and telescope quality as free parameters. From this, the optimum telescope diameter and surface quality are chosen as a function of performance. The result is a final cost versus performance curve for a single ground-based optical telescope. A schematic depicting the approach is given in Fig. 1.

\section{Analysis}

In recent years, several large telescope systems have been or are being built. It is from data on cost and performance of these telescopes that projections are based. The diameter and surface quality of the telescope determine the cost of the telescope. In Fig. 2, the costs of various systems have been graphed as a function of diameter for numerous values of surface quality (Ref. 1). In order to facilitate the formulation of an analytical model, straight line approximations have been drawn through points on the graph which correspond to telescopes of similar surface quality. For example, UAM, UKIRT, MMT, SMT and NNTT (see Table 1) all have a blur circle of approximately one arc sec. ${ }^{2}$ The line through MMD corresponds to telescopes of $10 \mathrm{arc} \mathrm{sec} .^{2}$ Finally, the solar collectors and radio telescopes correspond to about 5 arc min. ${ }^{2}$ As can be seen from the graph of Fig. 2, similar surface quality telescopes form parallel linear lines on the $\log / \log$ graph. As the diameter or surface quality increases, telescope cost also increases. An analytical expression which describes these lines can be formulated in the following form:

$$
C=\alpha D^{x}
$$

where $x$ and $\alpha$ are scaling factors, $D=$ diameter, and $C=$ cost.

Values for $x$ and $\alpha$ were numerically determined from Fig. 2. It was found that $x$, the slope of the parallel linear lines, varied from 2.4 to 2.8 , so $x=2.6$ was assumed as an average. This is consistant with the RF model which scales as $x=2.78$ (Ref. 2). $\alpha$, the $y$ intercept point, was then determined (using $x=2.6$ ) to scale as follows:

$$
\alpha=F^{0.94}\left(10^{6}\right) / 9.15
$$

\footnotetext{
${ }^{2}$ Aden Meindel and Marjorie Meindel, private communications.
}

where $F$ is the telescope resolution or angular blur spot size in arc sec. As an example, consider a 5-m telescope with a 10-arc sec angular blur spot. From Eqs. (1) and (2), the cost of such a telescope is found to be approximately 0.8 million dollars, which agrees reasonably well with the graph in Fig. 2. As a first-order approximation the surface quality given by the blur circle is equated to the field of view of the system limited by the size of the detector, assuming all the light collected is incident on the detector. This is a valid assumption since we are using the telescope as a "light bucket." It is to be noted that the total field of view of the telescope will be much larger. For example, the detector may be optomechanically moved within the total field of view to detect an instantaneous field of view. It is this field of view that is equated to the angular blur circle formed by the telescope and referred to throughout the text.

In order to determine the necessary telescope diameter and field of view, it was necessary to relate these parameters to performance of the resulting communication system. To do this, a reference $X$-band $(8.5 \mathrm{GHz}) \operatorname{link}^{3}$ was first established, which consisted of a $4.5-\mathrm{m}$ spacecraft antenna with a $10-\mathrm{W}$ $\mathrm{X}$-band transmitter $(25 \mathrm{~W}$ of raw spacecraft transmitter power) and a 1985 vintage 64-m X-band receiving system. Such a reference system is capable of $26-\mathrm{kbps}$ data transfer from Saturn (range $=10 \mathrm{AU}$ ). Then a strawman optical system was defined, which consisted of a $28-\mathrm{cm}$ spacecraft telescope, the same raw spacecraft transmitter power and the same link range. The specific parameters of the reference $X$-band and strawman optical systems are shown in Appendix A. Using manual and computer analysis tools, the performance of the strawman optical system was then calculated for various values of receiver diameter and field of view. The performance was quantified in terms of achievable data rate. Plots of achievable data rate versus telescope diameter and field of view are shown in Fig. 3. These values were then compared to $26 \mathrm{kbps}$ (the reference system) to determine communications performance gain. A typical achievable data rate example calculation is shown in Appendix B.

Both daylight conditions and moonless night conditions were addressed. However, only the daylight case is shown in Fig. 3. As can be seen from the curves, by combining a larger diameter with a smaller field of view, the system performance is increased. However, as previously observed in Fig. 2, increasing diameter and/or decreasing field of view (increasing surface quality) results in higher cost. Since the highest performance for the least cost is desired, the results of Figs. 2 and 3 must be coupled together and optinized.

\footnotetext{
${ }^{3}$ Layland, J. W., "Conceptual Technology-Capability-Cost Curves," JPL Interoffice Memorandum JWL-85-58, Nov. 11, 1985 (JPL internal document).
} 
Combining Eqs. (1) and (2) with Fig. 3, a third set of curves is obtained which directly relate cost and performance. Cost as a function of performance (measured by increase of data rate capability relative to the reference $X$-band system) for a $10-\mathrm{m}$ diameter telescope is graphed in Fig. 4. Here, telescope field of view is a parameter and, again for simplicity, only the daylight background case is shown. It can be observed that as higher surface quality (or narrower field of view) is required to obtain a given performance, the cost increases dramatically. A set of curves for $5-\mathrm{m}, 10-\mathrm{m}$, and $15-\mathrm{m}$ telescopes are graphed in Fig. 5. In this figure, both the daylight background and moonless night background cases are included. It can be observed that costs rise sharply as narrower field of view is required. However, it can also be observed that if a specific curve rises sufficiently, using a larger diameter telescope with a wider (poorer surface quality) field of view produces the same performance at a reduced cost. For example, consider 18-dB performance gain during daylight conditions. A $10-\mathrm{m}$ telescope producing such a performance gain would require a surface quality consistent with approximately a $2-\mu \mathrm{rad}$ telescope limited field of view and have a cost of around $\$ 80$ million. (Such a telescope would be comparable in size, quality and cost to the Keck telescope which is currently under construction.) However, a 15-m telescope with close to 15 times the surface error $(30-\mu \mathrm{rad}$ FOV) could provide equivalent communications performance gain for only $\$ 20$ million.

To obtain a single cost versus performance curve, the mean performance gain was selected between daytime and nighttime values for each of the three telescope diameters shown. The cost was then minimized for a given performance gain by optimizing over the telescope diameter. Further smoothing permits interpolation between the discrete values of diameter treated. Figure 6 summarizes the results of this process. The bracketed line regions of the curve correspond to regions where the identified telescope diameters are expected to apply. Regions between correspond to interpolated diameters. This final cost versus performance curve is estimated to be accurate to within $\pm 3 \mathrm{~dB}$.

One issue not examined in this article is that of arraying of smaller telescopes rather than using a single larger telescope. This is because neither the performance models for arraying nor the necessary instrumental costs of arraying are sufficiently well characterized to permit an informative comparison. However, it is believed that arraying will further reduce costs for a given level of performance. This is because arraying costs tend to increase linearly with array area (i.e., as the square of the effective single aperture diameter), whereas the cost model used herein for a single aperture telescope varies as the diameter to the 2.6 power. This issue will be the subject of future investigations.

\section{Concluding Remarks}

A model has been developed to relate the cost of an optical telescope to the resulting optical communications performance. This was done by relating available telescope cost data to a pair of parameters, the telescope diameter and a measure of telescope quality. These same quantities were used in a communications model context to quantize the communications performance gain relative to an $\mathrm{RF}$ reference system. The cost and performance were then related through equivalent values of telescope diameter and quality. The final cost model then resulted by picking the telescope diameter (and associated telescope quality) which minimized telescope cost for a given amount of performance improvement.

\section{References}

1. Meinel, Aden and Meinel, Marjorie, "Very Large Optics of the Future," Optics News, pp. 9-14, Mar. 1986.

2. Potter, P. D., Merrick, W., Ludwig, A., Large Antenna Apertures and Arrays for Deep Space Communications, Technical Report 32-848, Jet Propulsion Laboratory, Pasadena, CA, Nov. 1, 1965.

3. Lambert, S. A., et al., Design and Analysis Study of a Spacecraft Optical Transceiver Package, McDonnell Douglas Astronautics Co., Final Report under JPL contract No. 967061, August 19, 1985. 
Table 1. Acronyms used in text and figures

\begin{tabular}{ll}
\hline HST & Hubble Space Telescope \\
MMD & Millimeter Dish \\
MMT & Multimirror Telescope \\
NNTT & National New Technology Telescope \\
OAO & Orbiting Astronomical Observatory \\
& (Copernicus) \\
OPTRANSPAC & Optical Transceiver Package \\
SMT & Sub-Millimeter Telescope \\
UAM & University of Mexico \\
UKIRT & United Kingdom Infrared Telescope \\
VLA & Very Large Array \\
\hline
\end{tabular}




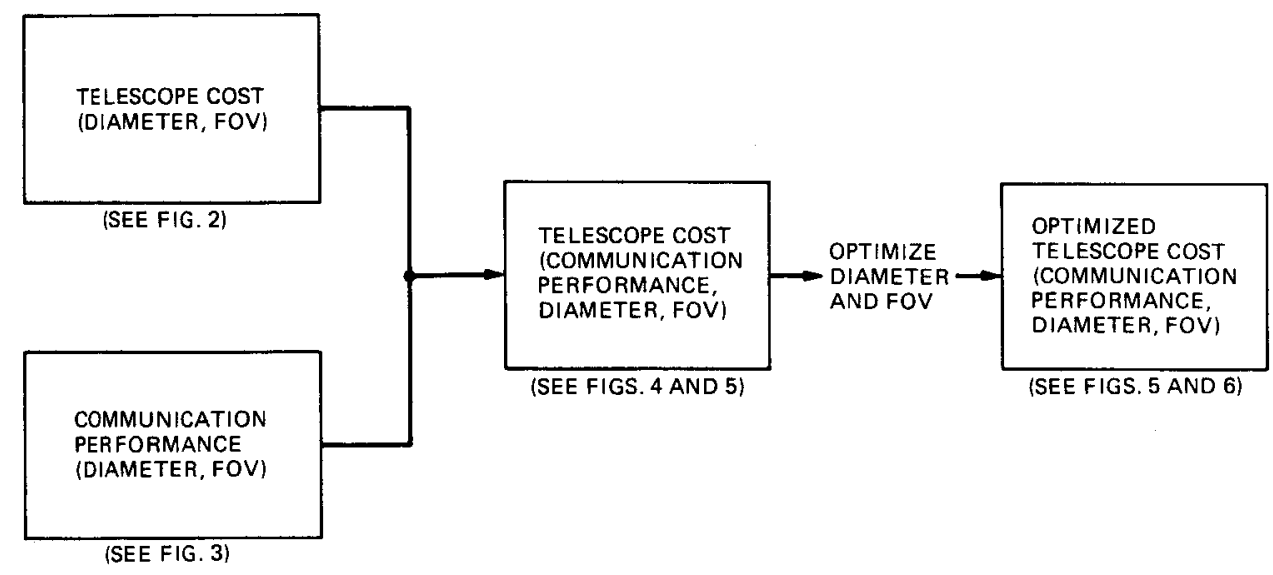

Fig. 1. Outline of approach for cost-performance modeling of optical communications receiving telescope

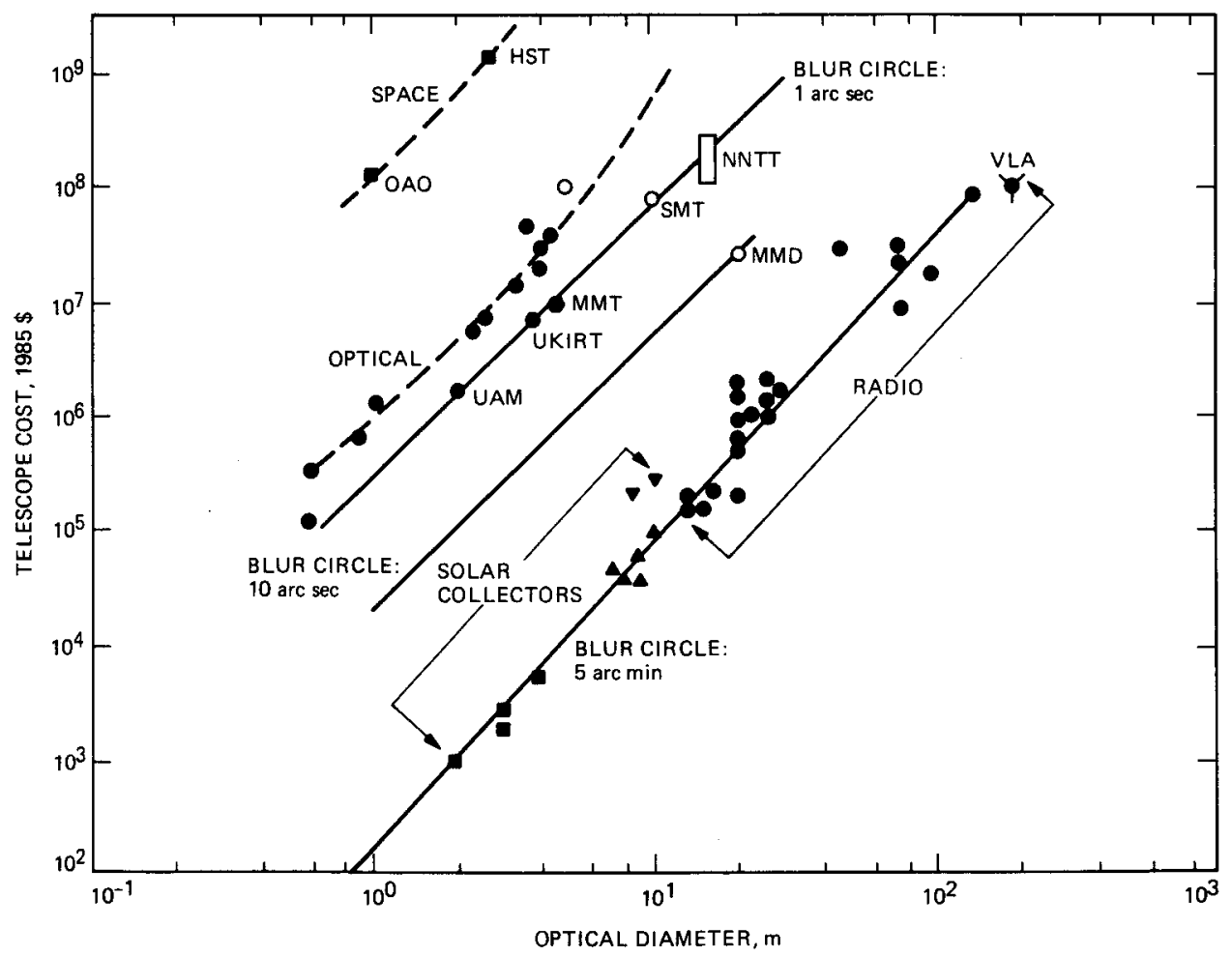

Fig. 2. Telescope costs as a function of diameter and angular blur circle resolution (see Table 1 for definition of acronyms) 


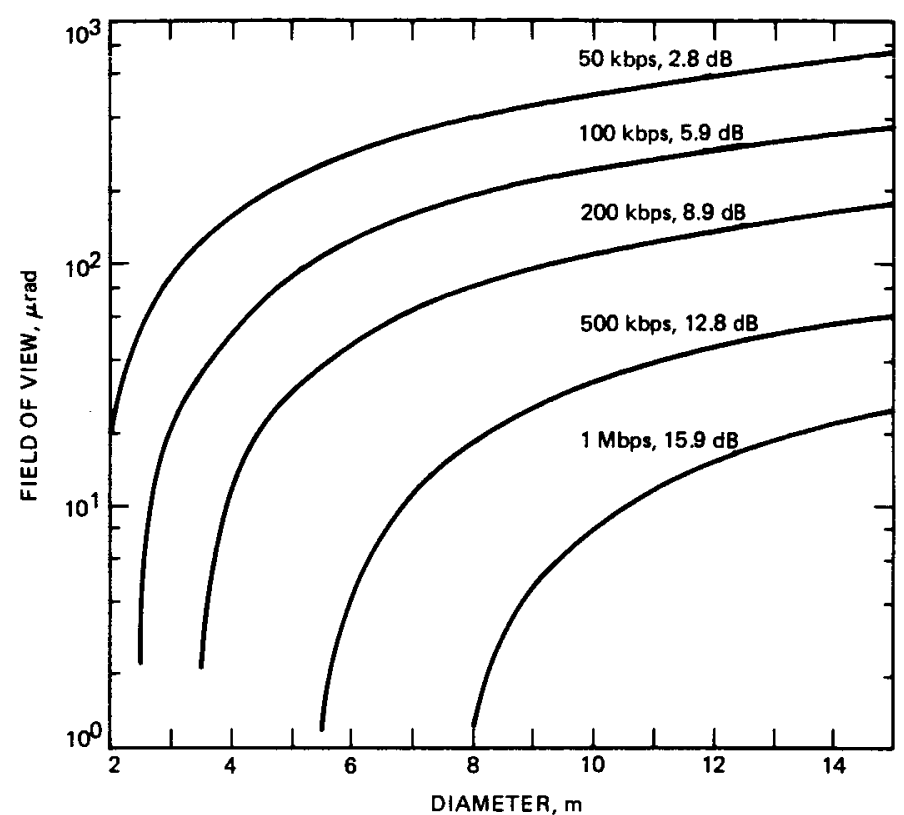

Fig. 3. Achievable optical communication data rates as a function of telescope diameter and field of view for daylight conditions (see Appendix A for specific operational characteristics)

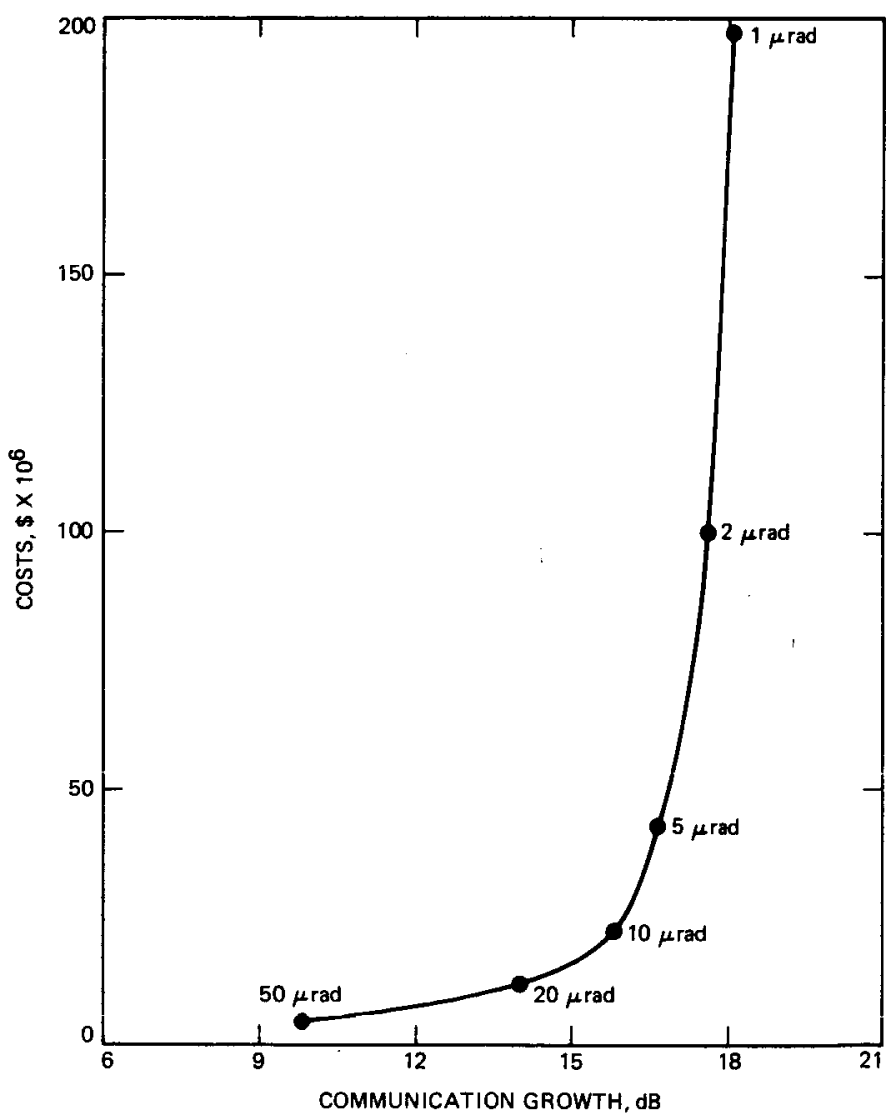

Fig. 4. Costs of 10-m telescope as a function of field of view and performance for daylight viewing conditions

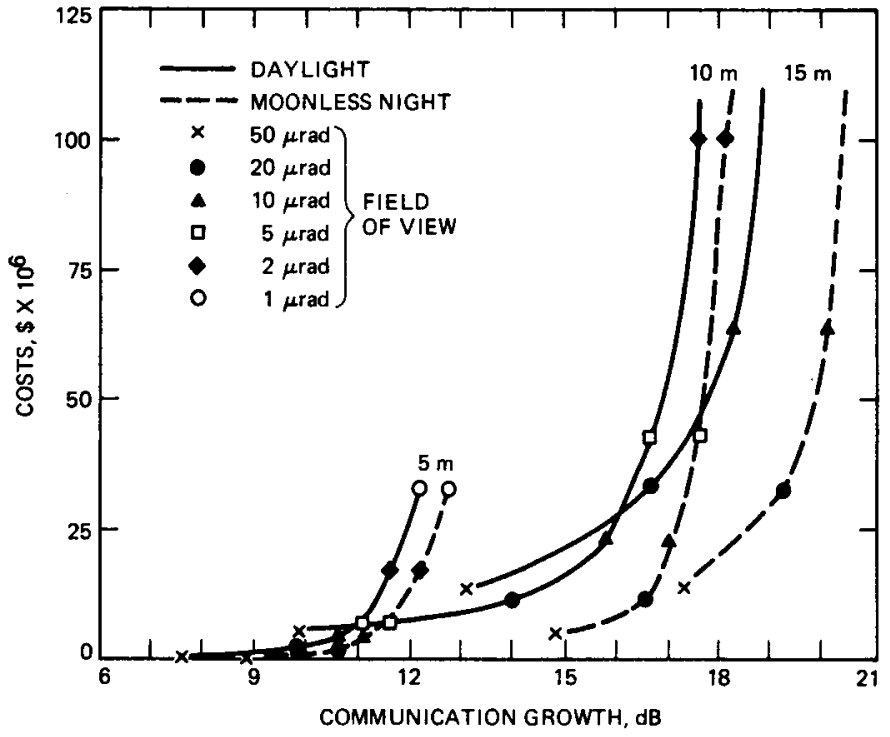

Fig. 5. Costs vs performance of 5-, 10, and 15-m telescopes for both daylight and moonless night conditions

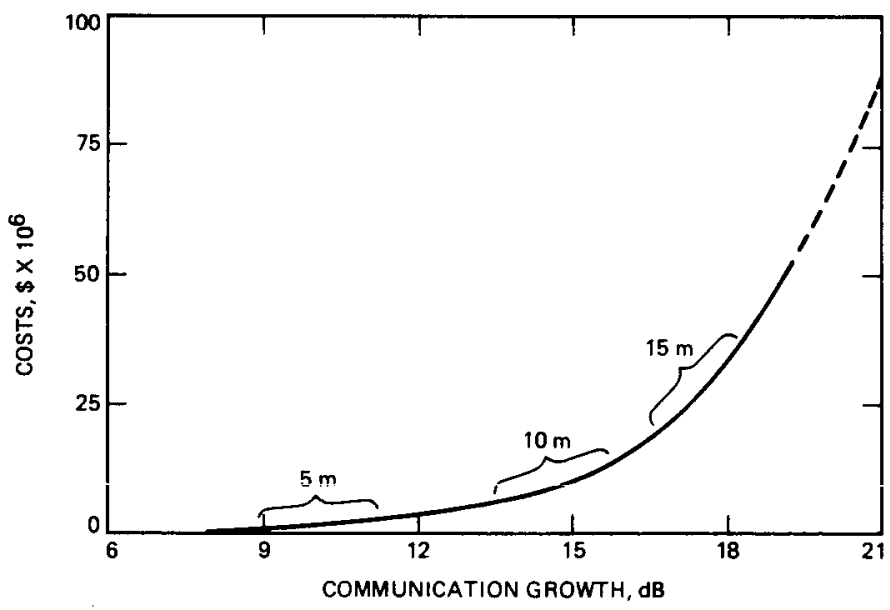

Fig. 6. Optimized telescope cost curve 


\section{Appendix A}

\section{Reference X-band $(8.5 \mathrm{GHz})$ and Strawman Optical System Description}

This appendix describes both the reference X-band $(8.5 \mathrm{GHz})$ system and the static portions of the strawman optical system used in this report.

\section{Reference X-band System}

The reference system against which optical communications performance was compared uses a $4.5-\mathrm{m}$ spacecraft antenna and a radio frequency wavelength of $3.5 \mathrm{~cm}$ (X-band). The spacecraft transmitter produces $10 \mathrm{~W}$ of X-band output power with a power conversion efficiency of $40 \%$. This gives rise to a $25 . \mathrm{W}$ overall power consumption of the transmitter. The communications range was from Saturn (10 AU), and the reception system was the 1985 version of a $64-m$ DSN station. This overall system is capable of achieving $26 \mathrm{kbps}$ from Saturn (see Footnote 3).

\section{Strawman Optical System}

The optical link considered consists of a $27.9-\mathrm{cm}$ telescope with a frequency doubled $\mathrm{Nd}$ :YAG laser at the spacecraft. The transmitted wavelength is $0.532 \mu \mathrm{m}$. The same raw transmitter power as the reference systems was assumed $(25 \mathrm{~W})$. This, coupled with an assumed laser power conversion efficiency, yields $2 \mathrm{~W}$ of laser power. The spacecraft was assumed to be at Saturn (10 AU range) with the planet directly behind the spacecraft. The values for transmitter diameter, transmitter obscuration $(0.0615 \mathrm{~m})$, transmitter pointing bias error $(0.3 \mu \mathrm{rad})$, and transmitter rms pointing jitter $(0.233 \mu \mathrm{rad})$ were taken from the OPTRANSPAC (Optical Transceiver Package) study (Ref. 3) performed by McDonnell Douglas. Atmospheric transmission, transmitter optics efficiency, receiver optics efficiency, and the quantum efficiency of the detector (with a spectral bandwidth of 10 angstroms) were each 50\%. The receiver diameter, field of view and data rate were variable parameters to be evaluated. Pulse position modulation with $M=256$, and a slot width of $10 \mathrm{~ns}$ were specified. The link performance was specified at an uncoded bit error rate of $10^{-3}$, a value which can be made much smaller than $10^{-6}$ with even a moderate amount of coding. 


\section{Appendix B \\ Sample Calculation of Optical Link Performance}

Antennae parameters:

Wavelength, $\mu \mathrm{m}$

Transmitted average power, $\mathrm{W}$

Distance between XMTR and RCVR, AU

Amospheric transmission fraction

Diameter of XMTR, $m$

Obscuration diameter of XMTR, $m$

XMTR optics efficiency

XMTR pointing bias error, $\mu \mathrm{rad}$

XMTR rms pointing jitter, $\mu$ rad

Diameter of RCVR, $m$

Obscuration diameter of RCVR, $m$

RCVR optics efficiency

Narrow band filter transmission

Spectral bandwidth, $\AA$

RCVR diam, field of view, $\mu \mathrm{rad}$

$=0.532$

$=2.00$

$=10.0$

$=0.500$

$=0.279$

$=0.615 \times 10^{-1}$

$=0.500$

$=0.300$

$=0.233$

$=10.0$

$=0.0$

$=0.500$

$=1.00$

$=10.0$

$=10.0$

PPM detector parameters:

Alphabet size $(M=$ ?)

Data rate, kbits/s

Dead time, $\mu \mathrm{s}$

Slot width, ns

Desired probability of bit error

Quantum efficiency of detector

$=256.0$

$=26.0$

$=305.0$

$=10.0$

$=1.0 \times 10^{-3}$

$=0.500$

Noise sources:

Saturn - RCVR to source distance, AU

$=10.0$

Additional noise sources:

DAYLIGHT - radiance, $\mathrm{W} / \mathrm{m}^{2} / \mathrm{sr} / \AA$

$=0.323 \times 10^{-2}$ 


\section{LINKOUTPUT}

\begin{tabular}{|c|c|c|c|}
\hline & & Factor & $\mathrm{dB}$ \\
\hline Transmitted power, $\mathrm{W}$ & & 2.00 & $33.0 \mathrm{dBm}$ \\
\hline Min Reqd peak power, $\mathrm{W}$ & $=0.62 \times 10^{5}$ & & \\
\hline XMTR antenna gain & & $0.187 \times 10^{13}$ & 122.7 \\
\hline XMTR diam, m & $=0.279$ & & \\
\hline Obscuration diam, $\mathrm{m}$ & $=0.062$ & & \\
\hline Beam width, $\mu \mathrm{rad}$ & $=3.328$ & & \\
\hline XMTR optics efficiency & & 0.500 & -3.0 \\
\hline XMTR pointing efficiency & & 0.878 & -0.6 \\
\hline Pointing error, $\mu \mathrm{rad}$ & $=0.300$ & & \\
\hline RMS jitter, $\mu \mathrm{rad}$ & $=0.233$ & & \\
\hline Space loss $(10.00 \mathrm{AU})$ & & $0.801 \times 10^{-39}$ & -391.0 \\
\hline Atmospheric transmission & & 0.500 & -3.0 \\
\hline RCVR antenna gain & & $0.349 \times 10^{16}$ & 155.4 \\
\hline RCVR diam, $\mathrm{m}$ & $=10.000$ & & \\
\hline Obscuration diam, $\mathrm{m}$ & $=0.000$ & & \\
\hline Field of view, $\mu \mathrm{rad}$ & $=10.000$ & & \\
\hline RCVR optics efficiency & & 0.500 & -3.0 \\
\hline Narrow band filter transmission & & 1.00 & 0.0 \\
\hline Bandwidth, $\AA$ & $=10.000$ & & \\
\hline Received signal power, $\mathrm{W}$ & & $0.114 \times 10^{-11}$ & $-89.4 \mathrm{dBm}$ \\
\hline Received noise power, $W$ & $=0.548 \times 10^{-10}$ & & \\
\hline Quantum efficiency & & 0.500 & -3.0 \\
\hline Photons/joule & & $0.268 \times 10^{19}$ & $184.3 \mathrm{~dB} / \mathrm{J}$ \\
\hline Received signal photoelectrons ( $\mathrm{F}$ & $\mathrm{PE}) / \mathrm{s}$ & $0.153 \times 10^{7}$ & $61.9 \mathrm{~dB} \cdot \mathrm{Hz}$ \\
\hline Symbol time, $s$ & & $0.308 \times 10^{-3}$ & $-35.1 \mathrm{~dB}-\mathrm{Hz}$ \\
\hline Received signal PE/symbol & & 472.0 & 26.7 \\
\hline Required signal PE/symbol & & 13.7 & 11.4 \\
\hline Received noise PE/slot & $=0.735$ & & \\
\hline Margin & & 34.6 & 15.3 \\
\hline
\end{tabular}

